\title{
LA ATENCIÓN A LA DIVERSIDAD EN EL AULA: DIFICULTADES Y NECESIDADES DEL PROFESORADO DE EDUCACIÓN SECUNDARIA Y UNIVERSIDAD
}

\author{
$M^{a}$ Emma Mayo Pais \\ Josefa del Carmen Fernández de la Iglesia \\ Felipe Roget Salgado \\ Universidad de Santiago de Compostela
}

RESUMEN: En el presente estudio se analizan las necesidades y dificultades para la atención educativa del profesorado a alumnado con discapacidad en el aula y su relación con distintas variables: sexo y edad del profesorado, tipo de discapacidad y etapa educativa. En relación con esta última variable se pretende comparar las reacciones del profesorado de secundaria con las del universitario, a través de una muestra de 200 docentes de ambos niveles educativos. Se ha observado, tras la aplicación de una adaptación del "Cuestionario para los profesores universitarios" (Castellana y Sala, 2005), que el profesorado de secundaria está más familiarizado con la presencia de alumnado con discapacidad y está más dispuesto a adaptar su metodología, pero a pesar de ello se muestra más inseguro que el universitario, y en consecuencia reclama una mayor formación, especialmente en los casos de discapacidad visual, así como un servicio de mediación y apoyo, que no parece identificarse con el departamento de Orientación. Estas necesidades no cubiertas podrían justificar la valoración mayoritariamente negativa de las experiencias con alumnado con discapacidad, especialmente en la Universidad, donde el desarrollo curricular se impone sobre el desarrollo personal.

PALABRAS CLAVE: inclusión, diversidad, discapacidad, actitudes.

\section{ATTENTION TO DIVERSITY IN THE CLASSROOM: DIFFICULTIES AND NEEDS OF HIGH SCHOOL AND UNIVERSITY TEACHERS}

\footnotetext{
ABSTRACT: In the present study the needs and difficulties of teachers to the presence of students with disabilities in the classroom and its relationship with different variables are analysed: sex and age of the teacher, type of disability and educational stage. In relation to this last variable, we intend to
} 
compare the reactions of secondary school teachers with those of university students, through a sample of 200 teachers from both educational levels. It has been observed, after applying an adaptation of the "Questionnaire for university professors" (Castellana and Sala, 2005), that secondary school teachers are more familiar with the presence of learners with disabilities and are more willing to adapt their methodology, but in spite of this, he is more insecure than the university student, and consequently he demands more training, especially in the case of students with visual disabilities, as well as a mediation and support service, which does not seem to be identified with the Orientation department. These unmet needs could justify the mostly negative evaluation of experiences with students with disabilities, especially in the University, where curricular development is imposed on personal development.

KEYWORDS: Inclusion, diversity, disability, attitudes.

Recibido: 11/04/2019

Aceptado: 07/06/2019

Correspondencia: $M^{a}$ Emma Mayo Pais, Facultad de Psicología, Universidad de Santiago de Compostela, Campus Vida, Calle Xosé María Suárez Núñez, s/n, 15782 Santiago de Compostela. Email: emma.mayo@usc.es.

\section{INTRODUCCIÓN}

La atención educativa a las personas con discapacidad ha experimentado muchos cambios a lo largo de los últimos años, sin embargo, la inclusión de alumnado con discapacidad en los centros ordinarios sigue siendo uno de los temas de debate más controvertidos en el ámbito educativo.

El punto de partida para la inclusión de estudiantes con discapacidad en la escuela ordinaria en España se sitúa en los años ochenta, con la Ley Social de Integración de los Minusválidos (LISMI, 1982), que sentó las bases para las futuras reformas de la legislación educativa relacionada con la escolarización del alumnado con necesidades específicas de apoyo educativo (N.E.A.E.) a través de la Ley General de Ordenación del Sistema Educativo (LOGSE, 1990), la Ley Orgánica de Calidad de la Educación (LOCE, 2002), la Ley Orgánica de Educación (LOE, 2006) y la actual Ley Orgánica de Mejora de la Calidad Educativa (LOMCE), vigente en España desde el año 2013. Sin embargo, a nivel universitario, la Ley de Reforma Universitaria (LRU), aprobada en 1983, no introdujo ningún aspecto relacionado con la atención a alumnado universitario con discapacidad. No fue hasta el año 2001 cuando la Ley Orgánica de Universidades (LOU) contempló en su disposición adicional vigésimo cuarta, la inclusión de las personas con discapacidad en las universidades, contemplando los principios de igualdad de oportunidades, no discriminación y accesibilidad universal de las personas con discapacidad. Sin em- 
bargo, la aplicación en los Estatutos de cada una de las universidades nacionales fue muy dispar y dilatada en el tiempo.

A pesar de los cambios legislativos producidos, el profesorado no dispone de recursos ni de herramientas para hacer viable una inclusión real, que precisa de una serie de adaptaciones y recursos de apoyo imprescindibles para el estudiantado que presenta discapacidad (Suriá, Bueno y Rosser, 2010).

Muchos han sido los factores relacionados con la inclusión. Por una parte se han señalado variables relacionadas con el alumnado como el tipo de discapacidad (Álvarez Martiño, Álvarez Hernández, Castro Pañeda, Campo Mon, y Fueyo Gutierrez, 2008; Avramidis, Bayliss y Burden, 2000).

Por otra parte, se han identificado variables relacionadas con el profesorado reconociendo que estos se identifican como un factor clave que ejerce gran influencia en el funcionamiento de la inclusión y de la atención al alumnado con necesidades especiales (Álvarez Martiño et al., 2008; Luque Espinoza, 2017; Palma et al., 2016). Las investigaciones muestran que, en general e independientemente del nivel educativo en el que imparte docencia, el profesorado reconoce importantes carencias formativas en la atención a alumnado con discapacidad (Avramidis et al., 2000; Castellana y Sala, 2005; Sánchez-Palomino, 2011), identificando el ámbito formativo como un elemento clave que incide en las actitudes del profesorado y que determina el éxito de la inclusión (Angenscheidt y Navarrete, 2017; Idol, 2006; Pegalajar y Colmenero, 2017).

Asimismo, se han señalado los años de docencia como variable a considerar. En este sentido, la literatura sobre el tema arroja resultados contradictorios discrepando los resultados de las distintas investigaciones entre aquellas que concluían que el profesorado más joven y con menos años de experiencia docente era el que mostraba actitudes más favorables hacia la inclusión (Alghazo y Naggar, 2004; Castellanos Daza, Gutiérrez Torres y Castañeda Polanco, 2018; Clavijo, López, Cedillo, Mora y Ortiz, 2016; Garzón, Calvo y Orgaz, 2016), las que hallaron que era el profesorado con mayor experiencia docente el que tenía actitudes más positivas hacia la inclusión (Angenscheidt y Navarrete, 2017) y las que no encontraban diferencias significativas en función de dicha variable (Avramidis et al., 2000; Garabal Barbeira, Pousasa, Espinosa, Canosa y Luis, 2018).

Se ha señalado que poseer experiencia previa, con alumnado con discapacidad, puede conllevar actitudes significativamente más favorables hacia la inclusión (Álvarez y Buenestado, 2015; Angenscheidt y Navarrete, 2017; Avramidis et al., 2000; Novo-Corti, Muñoz-Cantero y Calvo-Babío, 2015; Rodríguez-Martín y Álvarez-Arregui, 2015; Vaz et al., 2015) o actitudes ligeramente más positivas (Sánchez Palomino, 2011).

Respecto al género del profesorado tampoco hay datos concluyentes; algunos estudios revelaron actitudes más positivas por parte de las profesoras (Alghazo y Naggar, 2004; Álvarez y Buenestado, 2015; Avramidis et al., 2000; Sánchez-Palomino, 2011; Pegalajar y Colmenero, 2017; Vaz et al., 2015); mientras que otros no encontraron diferencias significativas (Álvarez y Buenestado, 2015; Clavijo et al., 2016; Garzón et al., 2016). 
Investigaciones anteriores mostraron que el nivel educativo correlaciona significativamente con una actitud positiva hacia la inclusión por parte del profesorado, siendo los de educación primaria los que muestran mejores actitudes (Garzón et al., 2016; Savage y Wienke, 1989).

Los cambios acaecidos en la atención educativa a alumnado con necesidades específicas de apoyo educativo se han producido, además, gracias a los avances tecnológicos y al cambio de las filosofías educativas, que han superado los planteamientos de la educación especial para centrarse en los de la inclusión en el sistema educativo ordinario. Todo ello, además, ha tenido consecuencias directas en el aumento de estudiantes con discapacidad en las aulas universitarias que se ha incrementado mucho a lo largo de los últimos años (Alcedo, Aguado, Real, González y Rueda, 2007; Castellana y Sala, 2005; De la Red, De la Puente, Gómez y Carro, 2002; GonzálezBadía y Molina, 2006).

La atención al alumnado desde una perspectiva inclusiva es un tema especialmente tratado y estudiado en el contexto de la educación primaria y secundaria pero apenas en el de los estudios universitarios (Castellana y Sala, 2005).

De esta forma, la presencia de este alumnado en los centros ha comportado importantes cambios organizativos, metodológicos y curriculares, así como una demanda insistente de recursos materiales, personales y de formación. En este sentido, los mayores obstáculos y dificultades que señala el profesorado para la aplicación de la inclusión educativa aluden a la falta de recursos materiales y personales, a la heterogeneidad de alumnado en los grupos de clase y a la falta de preparación para la atención a la diversidad.

Por otro lado, en el ámbito universitario las aulas no disponen de los recursos necesarios para favorecer la inclusión de este estudiantado y el profesorado no imparte sus asignaturas utilizando las metodologías adecuadas para que puedan participar en ellas (Castellana y Sala, 2005; Suriá et al., 2010). El profesorado ha de tener la oportunidad de formarse y sensibilizarse para comprender las ventajas de la inclusión, que empieza con una actitud de aceptación y disposición al cambio cuando es necesario.

Por tanto, independientemente del nivel educativo en el que imparte clase, el profesorado debe estar en un continuo período de formación y de actualización (Coelho, Blázquez y Cubo, 2017; Garzón et al., 2016; Luque Espinoza, 2017; RodríguezMartín y Álvarez-Arregui, 2015), de modo que este nivel de exigencia no derive en una repercusión de la existencia de problemas relacionados con su actitud hacia el alumnado con discapacidad.

Esto lleva a considerar al profesorado como un elemento clave para los procesos de inclusión, de forma que el objetivo de esta investigación es doble: por un lado, evaluar cuales son las necesidades y las dificultades del mismo ante la presencia de alumnado con discapacidad en sus aulas y, por otro, analizar las posibles diferencias existentes entre el profesorado de educación secundaria y universitaria en cuanto a la atención de estudiantado con discapacidad. 


\section{Método}

\section{Muestra}

La muestra estuvo formada por un total de 200 docentes de la Comunidad Autónoma de Galicia. De ellos, un 43\% $(n=86)$ eran hombres y un 57\% $(n=114)$ eran mujeres. Todos los sujetos tenían unas edades comprendidas entre los 26 y los 68 años, siendo el rango de edad de los 38-48 años el más frecuente (véase Figura 1).

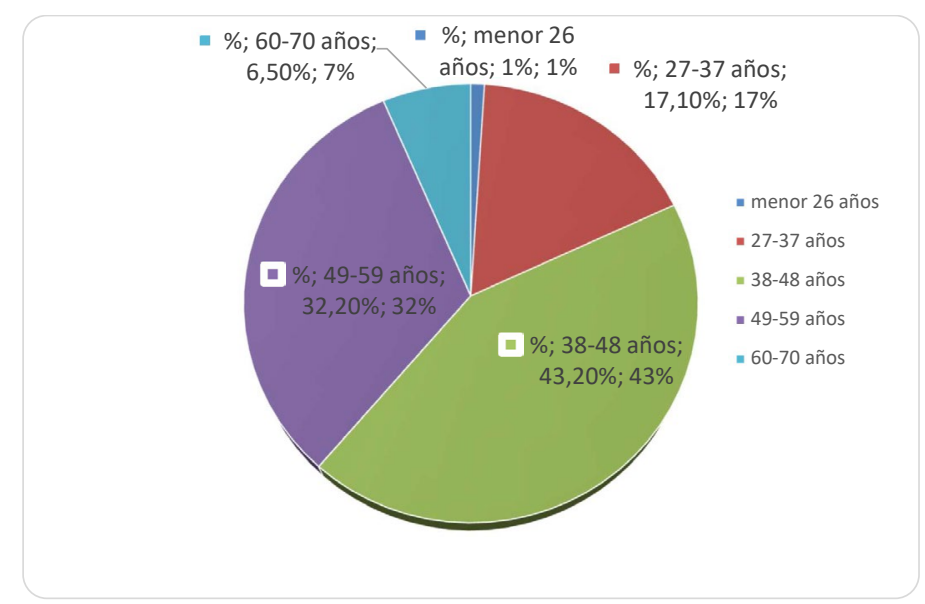

Figura 1. Distribución en porcentajes de la edad de la muestra

Los 100 docentes universitarios que han participado en la investigación trabajan en la Universidad de Santiago de Compostela (provincia de A Coruña), mientras que los demás docentes ejercen su actividad profesional en las cuatro provincias que conforman la Comunidad Autónoma de Galicia.

\section{Instrumento}

Para llevar a cabo el estudio se utilizó el "Cuestionario para los profesores universitarios" (Castellana y Sala, 2005) con pequeños ajustes en el planteamiento de las preguntas en el caso de profesorado de secundaria, para poder adaptarlo a la población a la que iba dirigido, tanto en el enunciado de los ítems como en el idioma utilizado (el Cuestionario se envió a todos los sujetos redactado en gallego, idioma co-oficial de la Comunidad Autónoma de Galicia).

Este instrumento ha permitido valorar lo que el colectivo de docentes expresa respecto a las necesidades y dificultades que tiene dentro del aula a la hora de impartir sus asignaturas.

El instrumento utilizado es un cuestionario semi-estructurado, con preguntas abiertas y cerradas, constituido por 15 ítems que recogen información acerca de las necesidades y 
dificultades de los profesores para atender al alumnado con distintos tipos de discapacidad en el aula ordinaria. Según sus propias autoras, en su elaboración se tuvieron en cuenta los siguientes aspectos, relacionados con la inclusión educativa: presencia de estudiantes con discapacidad en las aulas universitarias, metodología y formación del profesorado, conocimiento de las discapacidades y actitudes hacia los estudiantes con discapacidad.

\section{Procedimiento}

El envío del cuestionario se realizó a través de dos vías diferentes: en el caso de profesorado de secundaria, el envío se realizó a través del correo electrónico, de modo que se envió un correo a la Secretaría de los centros educativos de la Comunidad Autónoma de Galicia, solicitando su distribución al profesorado y la colaboración del mismo. En el caso del profesorado de la Universidad de Santiago de Compostela, se envió el cuestionario en formato papel utilizando, para ello, el servicio de correo interno de la citada Universidad.

En el proceso de investigación se han seguido los siguientes principios éticos:

- la participación ha sido totalmente voluntaria, de tal manera que son libres de negarse a participar y también pueden retirarse de la investigación en cualquier momento, sin ningún tipo de prejuicio para los participantes.

- Las personas que participaron en la investigación fueron informadas de los objetivos de la misma.

- Se ha garantizado la confidencialidad de los datos personales, respetando en todo momento la Ley de Protección de Datos Personales. Sobre esta base, se ha omitido toda la información que podría revelar la identidad de los sujetos. Además, se ha limitado el acceso a los datos (solo el equipo de investigadores puede ingresarlos en la base de datos) y todos los datos se han anonimizado.

\section{Análisis de datos}

Se han obtenido estadísticos descriptivos (frecuencias y porcentajes) para caracterizar las respuestas de los participantes del estudio a los distintos ítems del cuestionario, tanto para la muestra en conjunto como para cada grupo de interés (docentes de secundaria y docentes universitarios) por separado. Todas las variables dependientes (ítems del cuestionario) analizadas eran nominales u ordinales con 4 niveles de respuesta; por ello, se han llevado a cabo pruebas $\chi^{2}$ para contrastar si existía relación entre la variable independiente (nivel educativo en que imparte el docente) y las variables dependientes. Además, se han obtenido coeficientes de contingencia (estadístico Phi) para comprobar el grado o fuerza de relación entre dichas variables. Aunque se han realizado los mismos análisis para contrastar la posible relación entre las respuestas al cuestionario y las variables edad y género, no se han obtenido efectos significativos, por lo que dichos datos no se han incluido en la descripción de los resultados.

Todos los análisis estadísticos se han realizado con el paquete estadístico SPSS 20 para Mac (IBM, Inc.). 


\section{Resultados}

\section{Presencia de estudiantes con discapacidad dentro de las aulas de secundaria y universitarias}

A la pregunta " ¿ha tenido estudiantes con discapacidad en sus clases?" la mayoría de los profesores de la muestra (73\%) responden de forma afirmativa.

Los contrastes estadísticos han mostrado diferencias significativas en relación a la presencia de este alumnado en los diferentes niveles educativos $\left(\chi^{2}(1)=22.831, p \leq\right.$ .001 ; phi $=.34, \mathrm{p} \leq .001)$ siendo su presencia mayor en secundaria. En concreto, el $88 \%$ de los docentes de secundaria afirman haber tenido en alguna ocasión algún o alguna estudiante con discapacidad en el aula, frente al 58\% de los docentes universitarios (véase Tabla 1).

Tabla 1. Presencia de estudiantes con discapacidad en las aulas ordinarias y universitarias

\begin{tabular}{|l|c|c|c|c|c|}
\hline \multirow{2}{*}{\multicolumn{2}{|c|}{}} & \multicolumn{3}{|c|}{ ¿Ha tenido estudiantes con discapacidad en sus clases? } \\
\cline { 3 - 6 } \multicolumn{2}{|c|}{} & \multicolumn{2}{|c|}{ NO } & \multicolumn{2}{c|}{ Sí } \\
\cline { 3 - 6 } & $f$ & $\%$ & $f$ & $\%$ \\
\hline \multirow{2}{*}{ NIVEL EDUCATIVO } & Secundaria & 12 & 6.00 & 88 & 44.00 \\
\cline { 2 - 6 } & Universidad & 42 & 21.00 & 58 & 29.00 \\
\hline \multicolumn{2}{|l|}{ TOTAL } & $\mathbf{5 4}$ & $\mathbf{2 7 . 0 0}$ & $\mathbf{1 4 6}$ & $\mathbf{7 3 . 0 0}$ \\
\hline
\end{tabular}

\section{Evaluación del malestar por tener alumnado con discapacidad}

Con respecto a las respuestas del profesorado en cuanto al sentimiento de incomodidad/inseguridad suscitado por la presencia de alumnado con discapacidad en sus aulas, destaca un mayor nivel de malestar manifestado por el profesorado de secundaria $\left(\chi^{2}(3)=31.160, p \leq .001 ; p h i=.462, p \leq .001\right)$. Como se puede observar en la Tabla 2, un $15 \%$ de profesores de secundaria expresan haber sentido muchas veces o siempre incomodidad con dicha presencia, mientras que ningún docente universitario ha manifestado dichos niveles de malestar.

Tabla 2. Malestar ante la presencia de estudiantes con discapacidad en las aulas

\begin{tabular}{|l|l|c|c|c|c|c|c|c|c|}
\hline \multirow{2}{*}{} & \multicolumn{6}{c|}{$\begin{array}{c}\text { En caso de haber tenido estudiantes con discapacidad } \\
\text { en sus clases, ise sintió incómodo y/o inseguro? }\end{array}$} \\
\cline { 2 - 11 } & \multicolumn{2}{|c|}{ NUNCA } & $\begin{array}{c}\text { ALGUNA } \\
\text { VEZ }\end{array}$ & \multicolumn{2}{c|}{$\begin{array}{c}\text { MUCHAS } \\
\text { VECES }\end{array}$} & \multicolumn{2}{c|}{ SIEMPRE } \\
\cline { 2 - 10 } & $f$ & $\%$ & $f$ & $\%$ & $f$ & $\%$ & $f$ & $\%$ \\
\hline \multirow{2}{*}{ NIVEL EDUCATIVO } & Secundaria & 22 & 15.07 & 51 & 34.93 & 14 & 9.59 & 1 & 0.68 \\
\cline { 2 - 10 } & Universidad & 40 & 27.40 & 18 & 12.33 & 0 & 0.00 & 0 & 0.00 \\
\hline TOTAL & $\mathbf{6 2}$ & $\mathbf{4 2 . 4 7}$ & $\mathbf{6 9}$ & $\mathbf{4 7 . 2 6}$ & $\mathbf{1 4}$ & $\mathbf{9 . 5 9}$ & $\mathbf{1}$ & $\mathbf{0 . 6 8}$ \\
\hline
\end{tabular}


Dentro del grupo de profesores que no han tenido alumnado con discapacidad en sus aulas, el 50\% creen que no se sentirían incómodos/inseguros ante la presencia de estos estudiantes, mientras que el $31.48 \%$ tienen dudas respecto a cómo se sentirían en una situación que, hasta el momento, no han experimentado. No obstante, se observan diferencias estadísticamente significativas $\left(\chi^{2}(1)=4.908, p \leq .05\right.$; phi $=.364$, $\mathrm{p} \leq .05)$ en función de la variable nivel educativo en el que imparten docencia, dado que el $41.7 \%$ del profesorado de secundaria se sentiría incómodo/inseguro ante la presencia de alumnado con N.E.A.E. en el aula, mientras que solamente un $11.9 \%$ del profesorado universitario tendría esta sensación (véase Tabla 3).

Tabla 3. Malestar ante la posibilidad de tener estudiantes con discapacidad en las aulas

\begin{tabular}{|c|c|c|c|c|c|c|c|}
\hline & \multicolumn{6}{|c|}{$\begin{array}{l}\text { En caso de NO haber tenido estudiantes con discapa- } \\
\text { cidad en sus clases, ¿cree que se sentiría incómodo y/o } \\
\text { inseguro si los tuviese? }\end{array}$} \\
\hline & & \multicolumn{2}{|c|}{ Sí } & \multicolumn{2}{|c|}{ NO } & \multicolumn{2}{|c|}{ NO LO SÉ } \\
\hline & & $f$ & $\%$ & $f$ & $\%$ & $f$ & $\%$ \\
\hline \multirow{2}{*}{ NIVEL EDUCATIVO } & Secundaria & 5 & 9.26 & 4 & 7.41 & 3 & 5.55 \\
\hline & Universidad & 5 & 9.26 & 23 & 42.59 & 14 & 25.93 \\
\hline \multicolumn{2}{|l|}{ TOTAL } & 10 & 18.52 & 27 & 50.00 & 17 & 31.48 \\
\hline
\end{tabular}

\section{Formación y soporte del profesorado}

Se ha encontrado que la práctica totalidad del profesorado (89.12\%) utilizan algún tipo de metodología especial para poder atender a la diversidad dentro del aula, ya sea alguna vez (33.68\%), muchas veces (25.39\%) o siempre $(30.05 \%)$, frente a un $10.88 \%$ que manifiesta no utilizar nunca este tipo de metodología (véase Tabla 4). Resulta conveniente destacar, con respecto a este dato que, aunque parece que existe una sensibilidad especial en el profesorado con el fin de favorecer la inclusión educativa, se ha encontrado que existen diferencias significativas entre los grupos estudiados $\left(\chi^{2}(3)=49.004, p \leq .001 ;\right.$ phi $\left.=.504, p \leq .001\right)$. Así, los datos (véase Tabla 4) muestran un mayor porcentaje de docentes de universidad respecto a los de secundaria en las dos categorías extremas de este ítem, es decir, que nunca realizan cambios en sus metodologías docentes o que siempre realizan dichos cambios.

Tabla 4. Utilización de metodologías para atender a la diversidad dentro del aula

\begin{tabular}{|c|c|c|c|c|c|c|c|c|c|}
\hline & \multicolumn{8}{|c|}{$\begin{array}{c}\text { Cuando imparte clases, ¿utiliza algún tipo de metodo- } \\
\text { logía especial para poder atender a la diversidad dentro } \\
\text { del aula? }\end{array}$} \\
\hline & & \multicolumn{2}{|c|}{ NUNCA } & \multicolumn{2}{|c|}{$\begin{array}{c}\text { ALGUNA } \\
\text { VEZ }\end{array}$} & \multicolumn{2}{|c|}{$\begin{array}{l}\text { MUCHAS } \\
\text { VECES }\end{array}$} & \multicolumn{2}{|c|}{ SIEMPRE } \\
\hline & & $f$ & $\%$ & $f$ & $\%$ & $f$ & $\%$ & $f$ & $\%$ \\
\hline \multirow{2}{*}{ NIVEL EDUCATIVO } & Secundaria & 4 & 2.08 & 50 & 25.91 & 32 & 16.58 & 13 & 6.74 \\
\hline & Universidad & 17 & 8.80 & 15 & 7.77 & 17 & 8.81 & 45 & 23.32 \\
\hline \multicolumn{2}{|l|}{ TOTAL } & 21 & 10.88 & 65 & 33.68 & 49 & 25.39 & 58 & 30.05 \\
\hline
\end{tabular}


Una gran mayoría $(80.50 \%)$, considera que es necesario recibir formación específica para atender a la diversidad en el aula y sólo un $6.50 \%$ afirma no necesitarla (véase Tabla 5). Nuevamente, se han encontrado diferencias estadísticamente significativas entre los diferentes grupos estudiados $\left(\chi^{2}(2)=20.297, p \leq\right.$ .001 ; phi $=.319, \mathrm{p} \leq .001)$, siendo los profesores de secundaria los que más parecen necesitar esa formación $(47 \%$, por un $33.5 \%$ en el caso de los docentes universitarios).

Tabla 5. Necesidad de formación específica

\begin{tabular}{|c|c|c|c|c|c|c|c|}
\hline & \multicolumn{6}{|c|}{$\begin{array}{l}\text { ¿Cree que los profesores tendrían que recibir alguna } \\
\text { formación específica para poder atender a la diversi- } \\
\text { dad dentro de las aulas? }\end{array}$} \\
\hline & & \multicolumn{2}{|c|}{ sí } & \multicolumn{2}{|c|}{ NO } & \multicolumn{2}{|c|}{ NO LO SÉ } \\
\hline & & $f$ & $\%$ & $f$ & $\%$ & $f$ & $\%$ \\
\hline \multirow{2}{*}{ NIVEL EDUCATIVO } & Secundaria & 94 & 47.00 & 2 & 1.00 & 5 & 2.50 \\
\hline & Universidad & 67 & 33.50 & 11 & 5.50 & 21 & 10.50 \\
\hline \multicolumn{2}{|l|}{ TOTAL } & 161 & 80.50 & 13 & 6.50 & 26 & 13.00 \\
\hline
\end{tabular}

Los resultados obtenidos con respecto al conocimiento específico para realizar adaptaciones metodológicas en función de los distintos tipos de discapacidad fueron los siguientes:

- Discapacidad visual: un 64.28\% manifiesta no saber realizar una adaptación metodológica de su asignatura en el caso de que hubiese una persona con deficiencia visual en su clase, sin que existan diferencias estadísticamente significativas entre los dos grupos analizados (véase Tabla 6).

- Discapacidad auditiva: el 51.55\% manifiestan desconocer cómo llevar a cabo dicha adaptación (véase Tabla 6). No obstante, se ha encontrado que existen diferencias estadísticamente significativas entre ambos grupos $\left(\chi^{2}(2)=13.021\right.$, $\mathrm{p} \leq .001$; phi $=.259, \mathrm{p} \leq .001)$, siendo el profesorado universitario el que más preparado se siente de cara a adaptar sus materias para alumnado con este tipo de discapacidad (véase Tabla 6).

- Discapacidad física: la mayoría de los participantes (69.31\%) manifiestan saber llevar a cabo dicha adaptación, sin que existan diferencias estadísticamente significativas entre los dos grupos analizados (véase Tabla 6).

Por otro lado, conviene destacar que se ha encontrado una relación estadísticamente significativa entre saber realizar una adaptación metodológica de la asignatura y un menor sentimiento de malestar (incomodidad/inseguridad) en los casos de discapacidad auditiva $\left(\chi^{2}(6)=14.681, \mathrm{p} \leq .05 ; \mathrm{phi}=.324, \mathrm{p} \leq .05\right)$ y de discapacidad física/motora $\left(\chi^{2}(3)=10.807, p \leq .01 ;\right.$ phi $\left.=.279, p \leq .01\right)$, aunque en el caso de la discapacidad visual no se ha encontrado esta relación. 
Tabla 6. Conocimiento sobre la realización de adaptaciones para alumnos con discapacidad visual, auditiva y motora

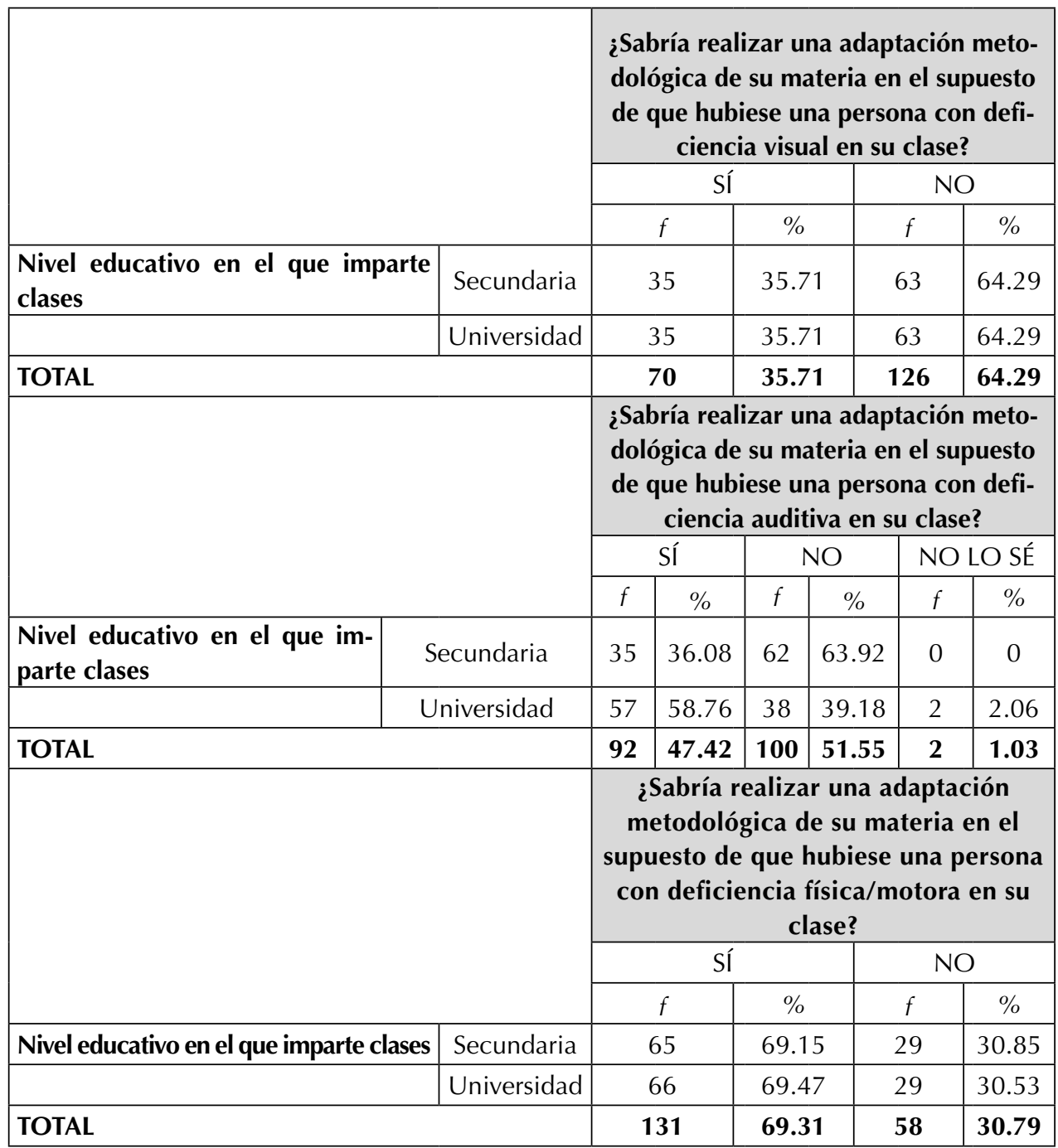

Para finalizar este bloque de "formación y soporte", indicar que el 59.50\% de la muestra apoya la idea de incorporar un servicio de mediación entre el estudiante con discapacidad y el profesorado y tan sólo un $14.0 \%$ dice que este servicio no sería necesario, sin que existan diferencias estadísticamente significativas entre los diferentes grupos estudiados (véase Tabla 7). 
Tabla 7. Necesidad de servicio de mediación entre estudiante con discapacidad y profesor

\begin{tabular}{|l|c|c|}
\hline & Frecuencia & Porcentaje \\
\hline SÍ & 119 & $\mathbf{5 9 . 5 0}$ \\
\hline NO & 28 & $\mathbf{1 4 . 0 0}$ \\
\hline NO LO SÉ & 53 & $\mathbf{2 6 . 5 0}$ \\
\hline TOTAL & $\mathbf{2 0 0}$ & $\mathbf{1 0 0 . 0 0}$ \\
\hline
\end{tabular}

\section{VAlORACIÓN DEL PROFESORADO SOBRE SU EXPERIENCIA EN ATENCIÓN A LA DIVERSIDAD}

Finalmente, en el cuestionario, se les preguntó a los profesores sobre la valoración de su experiencia cuando imparten clases a alumnado con discapacidad integrados en las aulas de secundaria y universitarias. El porcentaje de profesores que valora como negativa (deficiente y regular) su experiencia con estudiantes con N.E.A.E. $(75.35 \%)$ es superior a los que la consideran positiva $(24.65 \%)$ (Véase Tabla 8).

Tabla 8. Valoración de la experiencia

\begin{tabular}{|c|c|c|c|c|c|c|c|c|c|}
\hline & \multicolumn{8}{|c|}{$\begin{array}{l}\text { En caso de haber tenido estudiantes con discapacidad } \\
\text { en sus clases, ¿qué valoración general hace de esta } \\
\text { experiencia? }\end{array}$} \\
\hline & & \multicolumn{2}{|c|}{ DEFICIENTE } & \multicolumn{2}{|c|}{ REGULAR } & \multicolumn{2}{|c|}{ BUENA } & \multicolumn{2}{|c|}{ MUY BUENA } \\
\hline & & $f$ & $\%$ & $f$ & $\%$ & $f$ & $\%$ & $f$ & $\%$ \\
\hline \multirow{2}{*}{ NIVEL EDUCATIVO } & Secundaria & 19 & 13.02 & 36 & 24.66 & 21 & 14.38 & 12 & 8.22 \\
\hline & Universidad & 52 & 35.62 & 3 & 2.05 & 3 & 2.05 & 0 & 0.00 \\
\hline \multicolumn{2}{|l|}{ TOTAL } & 71 & 48.64 & 39 & 26.71 & 24 & 16.43 & 12 & 8.22 \\
\hline
\end{tabular}

Además, se han encontrado diferencias significativas $\left(\chi^{2}(1)=23.345, p \leq .001\right.$; phi $=.361, p \leq .001)$ entre la valoración realizada por los profesores de secundaria y la valoración realizada por los universitarios, siendo estos últimos los que peor han valorado la experiencia (véase Figura 2).

Para finalizar este apartado de resultados, conviene destacar que se ha hallado una relación significativa entre sentirse incómodo/inseguro y la valoración de la experiencia $\left(\chi^{2}(3)=179, \mathrm{p} \leq .001\right.$; phi $\left.=1.000, \mathrm{p} \leq .001\right)$, de modo que a mayor incomodidad/inseguridad manifestada por los docentes se obtienen valoraciones de la experiencia más negativas. 


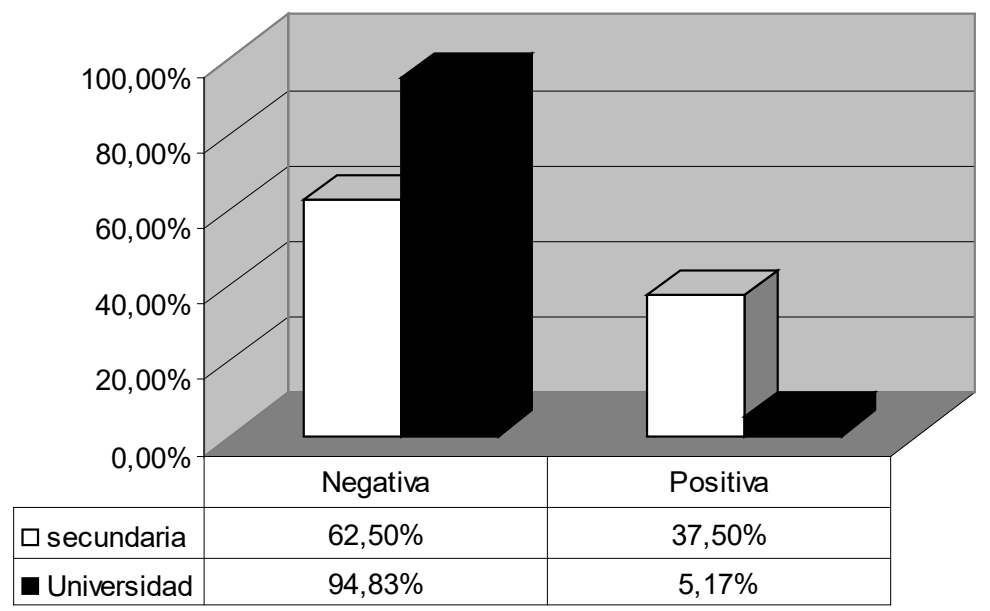

Figura 2. Valoración de la experiencia por nivel educativo

\section{Conclusiones y Discusión}

De manera global, los datos obtenidos en este estudio revelan que la presencia de alumnado con discapacidad en las aulas, tanto de secundaria como de universidad, es una realidad. No obstante, se ha constatado que, tal como se refleja en el Libro Blanco sobre Universidad y Discapacidad (Fundación Universia, 2016; Peralta, 2007), la presencia de dicho alumnado en los niveles superiores del Sistema Educativo es todavía escasa en relación a la educación secundaria. Esto puede deberse a que la educación del alumnado con necesidades educativas especiales está totalmente regulada a través de la Ley Orgánica 1/1990, de 3 de octubre, de Ordenación General del Sistema Educativo (LOGSE) que constituye, en nuestro ordenamiento jurídico, el punto de consolidación definitiva de los principios pedagógicos de integración y normalización educativas (art. 36.3). Así, en la Ley de Educación (LOE, art. 74.1) se puede observar toda una declaración de intenciones acerca de la inclusión de alumnado con necesidades educativas especiales en el aula ordinaria, si bien la ley actual (LOMCE) nos ha alejado de nuevo de un modelo de escuela comprensivo e inclusivo (López Melero, 2016). A nivel universitario el marco legislativo se encuentra en la actualidad menos desarrollado, produciéndose un importante aumento de las disposiciones legales, normativas y reglamentos que persiguen la plena inclusión de estos estudiantes, dirigiéndose todos los esfuerzos hacia la regulación de los principios de igualdad de oportunidades e inclusión de las personas con discapacidad en la vida académica universitaria.

Por otro lado, se ha encontrado que la mayor parte del profesorado no sabe realizar adaptaciones metodológicas de sus asignaturas para el alumnado con discapacidad (Aranda-Redruello, 2014) y que esta falta de preparación repercute de forma negativa en su labor docente, experimentando un cierto nivel de malestar (in- 
comodidad/inseguridad). Estos resultados coinciden con los aportados por GómezRestrepo, Rodríguez, Padilla y Avella-García (2009), que reflejan que a un 19.5\% del profesorado de su población de estudio no les agrada tener alumnado con discapacidad en sus aulas y más del $70 \%$ indicó no sentirse preparado para educar a estudiantes con algún tipo de discapacidad (mental, motora y sensorial). En esta línea, se han relacionado los factores ciclo educativo y niveles de estrés y malestar de los docentes, de manera que a mayor nivel educativo mayor nivel de estrés (Luque y Rodríguez, 2008). En función de esto, cabría esperar que el profesorado universitario fuese el que experimentase más malestar ante la presencia de alumnado con discapacidad en sus aulas, aunque los resultados obtenidos son justo los contrarios y coinciden con los encontrados por Suriá (2012). Este hecho podría ser explicado porque la carga docente en secundaria, con respecto a la formación del alumnado, sería mayor pues, aunque el colectivo de alumnado con discapacidad es cada vez más diverso y heterogéneo, si los comparamos con los de secundaria observamos que los universitarios disponen, a priori, de un mayor grado de autonomía en su aprendizaje, además de presentar un mayor grado de interés y de motivación que los de secundaria (Castellana y Sala, 2005).

Los resultados indican que la edad no influye en la percepción sobre el nivel de malestar del profesorado de acuerdo con investigaciones anteriores que no encontraron diferencias significativas en función de esta variable (Avramidis et al., 2000).

Con respecto al sexo, no se hallaron diferencias significativas en cuanto a la percepción de malestar. Los resultados no coinciden con los aportados en otras investigaciones, que indican que las mujeres tienen actitudes más positivas hacia la inclusión de alumnado con discapacidad (Avramidis et al., 2000; Novo-Corti et al., 2015; Sánchez-Palomino, 2011).

A pesar de todo lo anterior, se ha encontrado que existe una buena disposición por parte del profesorado para atender a este tipo de alumnado coincidiendo con estudios anteriores (Al-Busaidi y Tuzlukova, 2018; Avramidis y Kalyva, 2007; Castellana y Sala, 2005; Garzón et al., 2016; Ocampo-González, 2012; Polo, 2017; Sánchez-Palomino, 2011), aunque reclaman la necesidad de una mayor formación para poder dar una educación de calidad a su alumnado y consideran necesaria la presencia de un servicio de mediación especializado, cuyas funciones serían "asesorar al profesor en su labor docente"; "actuar como mediador entre el estudiante y el profesor, cuando la comunicación entre ambos no sea posible"; "garantizar los derechos del alumnado con discapacidad"; "evaluar el grado de éxito del profesor con ese alumno", etc. Este dato Ilama la atención, pues no existen diferencias significativas entre los diferentes niveles analizados, a pesar de que todas las funciones que los profesores de secundaria atribuyen a este "servicio de mediación especializado" son funciones propias de los Departamentos de Orientación regulados legislativamente, con lo que cabría esperar que los docentes de secundaria identificasen al Departamento de Orientación como "servicio de mediación especializado". No obstante, resulta conveniente destacar que este resultado ya ha sido obtenido en investigaciones anteriores (Mayo, Taboada, Iglesias-Souto y Van, 2008). 
Con respecto a la valoración que el profesorado hace de su experiencia en atención a la diversidad, se ha obtenido que el profesorado universitario es el que peor valora esta experiencia. Estos datos van en la línea de los obtenidos en anteriores estudios (Castellana y Sala, 2005), que concluyen que a medida que aumenta la edad de los estudiantes, la actitud del profesorado va siendo menos positiva, lo cual se atribuye a que los docentes de cursos superiores están más preocupados por el contenido de sus materias y menos por las diferencias individuales en el alumnado.

Como puede observarse, las necesidades y dificultades del profesorado de cara a la atención de alumnado con discapacidad son las mismas, independientemente del nivel educativo en el que se encuentren, si bien existen diferencias significativas que podríamos atribuir a cuestiones legislativas e, incluso, al diferente grado de madurez educativa de los estudiantes a los que los distintos docentes tienen que enfrentarse.

Para concluir, se puede afirmar que es necesario profundizar en este tema, pues para lograr la plena inclusión de alumnado con discapacidad se debe conocer la opinión del profesorado para poder atender, formar y resolver los conflictos que le puedan surgir en su labor como docentes. Así, los resultados obtenidos indican que se debe trabajar en dos líneas: por un lado, incrementar la formación docente con respecto a la discapacidad con el fin de disminuir los niveles de malestar docente y proporcionar una educación de calidad a este colectivo de alumnado. En línea con investigaciones anteriores que destacan la importancia de la formación para la generación de actitudes positivas hacia la inclusión (Álvarez-Pérez y López-Aguilar, 2014; Avramidis et al., 2000; Clavijo et al., 2016; Garabal Barbeira et al., 2018; Rodríguez-Martín y ÁlvarezArregui, 2015), que han reforzado la idea de que la formación en educación especial que se adquiere en cursos previos o coincidentes con la docencia se asocia a una menor resistencia frente a las prácticas inclusivas. Por otro lado, se deben proporcionar servicios de apoyo consolidados, con presupuestos estables, que presten una atención integral y multidisciplinar y que sean los encargados de impulsar medidas de accesibilidad, proporcionar productos de apoyo o asesoramiento docente, entre otras acciones.

A pesar de todo, no se debe pasar por alto algunas limitaciones del estudio que conciernen a la metodología del mismo, como es el tamaño reducido de la muestra. Por tanto, sería conveniente seguir investigando con una muestra más amplia en cuanto al número de docentes y ciclos educativos con el fin de poder plantear programas de mejora que permitan evitar el sentimiento de inseguridad/incomodidad que, de algún modo, va a influir en la calidad del ejercicio profesional, repercutiendo negativamente en el propio alumnado, en el proceso de enseñanza-aprendizaje e, inevitablemente, en la inclusión de los mismos. Además, como línea futura de investigación, está determinar las causas por las que el profesorado de secundaria no identifica al Departamento de Orientación como "servicio de medicación especializado".

\section{REFERENCIAS BIBLIOGRÁFICAS}

Al-Busaidi, S. y Tuzlukova, V. (2018). Teachers' Perceptions of Practices and Challenges of Innovating for the Inclusion of Special Needs University English Language Learners in Oman. Journal af Educational \& Psychological Studies, 14(4), 659-671. 
Alcedo, M.A., Aguado, A.L., Real, S., González, M. y Rueda, B. (2007). Una revisión de la situación de los estudiantes con discapacidad en la Universidad, Annuary of Clinical of Health Psychology, 3, 7-18.

Alghazo, E. y Naggar, E. (2004). General education teachers in the United Arab Emirates and their acceptance of the inclusion of students with disabilities. British Journal of Special Education, 31, 94-99.

Álvarez, J. L. y Buenestado, M. (2015). Predictores de las actitudes hacia la inclusión de alumnado con necesidades educativas especiales en futuros profesionales de la educación. Revista Complutense de Educación, 26(3), 627-645.

Álvarez Martiño, E. Álvarez Hernández, M., Castro Pañeda, P., Campo Mon, M. A. y Fueyo Gutierrez, E. (2008). Funcionamiento de la integración en la Enseñanza Secundaria Obligatoria según la percepción del profesorado. Psicothema, 20, 56-61.

Álvarez-Pérez, P. R. y López-Aguilar, D. (2014). Atención del profesorado universitario a estudiantes con necesidades educativas específicas. Educación y Educadores, 18(2), 193-208. http://doi.org/10.5294/edu.2015.18.2.1.

Angenscheidt, L. y Navarrete, I. (2017). Actitudes de los docentes acerca de la educación inclusiva. Ciencias Psicológicas, 11(2), 233-243.

Aranda-Redruello, R. (2014). La formación del profesorado universitario: mejora de los procesos de enseñanza-aprendizaje del alumnado con discapacidad. Tendencias Pedagógicas, 23, 271-300.

Avramidis, E. y Kalyva, E. (2007). The influence of teaching experience and professional development on Greek teachers' attitudes towards inclusion. European Journal of Special Needs Education, 22, 367-289.

Avramidis, E., Bayliss, P. y Burden, R. (2000). Inclusion in action: an in-depth 144 case study of an effective inclusive secondary school in the south-west of England. International Journal of Inclusive Education, 6, 38-43.

Castellana, M. y Sala, I. (2005). La Universidad ante la diversidad en el aula. Aula Abierta, 85, 57-84.

Castellanos Daza, C., Gutiérrez Torres, A. y Castañeda Polanco, J. (2018). Actitudes hacia la discapacidad en educación superior. Inclusión y Desarrollo, 5(2), 159174. https://doi.org/10.26620/uniminuto.inclusion.5.2.2018.159-174.

Clavijo, R., López, C., Cedillo, C., Mora, C. y Ortiz, W. (2016). Actitudes docentes hacia la educación inclusiva en Cuenca. MASKANA, 7(1), 13-22. https://doi. org/10.18537/mskn.07.01.02.

Coelho, F., Blázquez, F. y Cubo, S. (2017). Teacher training, attitudes and inclusion. International Journal of Technology and Inclusive Education, 6(1), 1032-1040.

De la Red, N., de la Puente, R., Gómez, M.C. y Carro, L. (2002). El acceso a los estudios superiores de las personas con discapacidad física y sensorial. Valladolid: Secretariado de Publicaciones e Intercambio Editorial. 
Fundación Universia (2016). Universidad y discapacidad: III estudio sobre el grado de inclusión en el sistema universitario español respecto de la realidad de la discapacidad. CERMI. Recuperado de http://riberdis.cedd.net/ bitstream/handle/11181/5199/Universidad_y_discapacidad_III_Estudio. pdf? sequence $=1$.

Garabal Barbeira, J., Pousasa, T., Espinosa, P., Canosa, S. y Luis, J. (2018). Las actitudes como factor clave en la inclusión universitaria. Revista Española de Discapacidad, 6(1), 181-198.

Garzón, P., Calvo, M.I. y Orgaz, M.B. (2016). Inclusión educativa. Actitudes y estrategias del profesorado. Revista Española de Discapacidad, 4 (2): 25-45. https://doi. org/10.5569/2340- 5104.04.02.02.

Gómez-Restrepo, C., Rodríguez, V., Padilla A. y Avella-García, C. (2009). El docente, su entorno y el síndrome de agotamiento profesional (SAP) en colegios públicos en Bogotá. Revista Colombiana de Psiquiatría, 38, 279-293.

González-Badía, J. y Molina, C. (2006). Situación de los estudiantes con discapacidad en las universidades españolas. Fiapas, 111, 30-33.

Idol, L. (2006). Toward Inclusion of Special Education Students in General Education. A Program Evaluation of Eight Schools. Remedial and Special Education, 27, 7794.

LISMI (1982). Ley Orgánica 13/1982, de 7 de abril, de Integración Social de los Minusválidos (BOE, de 10 de abril de 1982).

LOCE (2002). Ley Orgánica 10/2002, de 23 de diciembre, de Calidad de la Educación (BOE, de 24 de diciembre de 2002).

LOE (2006). Ley Orgánica 2/2006 de 3 de mayo, de Educación (B.O.E. de 4 de mayo de 2006).

LOGSE (1990). Ley Orgánica 1/1990 de 3 de octubre, de Ordenación General del Sistema Educativo. (B.O.E. de 4 de octubre de 1990).

LOMCE (2013). Ley Orgánica 8/2013, de 9 de diciembre, para la mejora de la calidad educativa. (B.O.E. de 10 de diciembre de 2013).

López Melero, M. (2016). La LOMCE, un cuadro tenebroso que no responde a la diversidad. Revista Educación, Política y Sociedad 1(2), 163-182. Recuperado de http://hdl.handle.net/10486/674645.

LOU (2001). Ley Orgánica 6/2001, de 21 de diciembre, de Universidades (BOE, de 24 de diciembre de 2001).

LRU (1983). Ley Orgánica 11/1983, de 25 de agosto, de Reforma Universitaria (BOE, de 1 de septiembre de 1983).

Luque Espinoza, M. P. (2017). Educación inclusiva. Experiencias docentes en la Universidad Metropolitana de Guayaquil. Revista de Educación Inclusiva, 10(1), 4558. 
Luque, D. J. y Rodríguez, G. (2008). Alumnado Universitario con Discapacidad: Elementos para la reflexión psicopedagógica. Revista Española de Orientación y Psicopedagógica, 19, 270-281.

Mayo, M. E., Taboada, E. M., Iglesias-Souto, P. M. y Van, E. (abril, 2008). La atención a la diversidad en el aula: necesidades y dificultades del profesorado. Comunicación presentada en el V Congreso Internacional de Psicología y Educación: los retos del futuro, Oviedo, España.

Novo-Corti, I., Muñoz-Cantero, J. M. y Calvo-Babío, N. (2015). Los futuros docentes y su actitud hacia la inclusión de personas con discapacidad. Una perspectiva de género. Anales de Psicología, 31(1), 155-171.

Ocampo-González, A. (2012). Inclusión de estudiantes en situación de discapacidad a la educación superior. Desafíos y oportunidades. Revista Latinoamericana de Educación Inclusiva, 6(2), 227-239.

Palma, O., Soto, X., Barría, C., Lucero, X., Mella, D., Santana, Y. y Seguel, E. (2016). Estudio cualitativo del proceso de adaptación e inclusión de un grupo de estudiantes de educación superior con discapacidad de la Universidad de Magallanes. Magallania (Punta Arenas), 44(2), 131-158.

Pegalajar, M. C. y Colmenero, M. J. (2017). Actitudes y formación docente hacia la inclusión en Educación Secundaria Obligatoria. Revista Electrónica de Investigación Educativa, 19(1), 84-97. https://doi.org/10.24320/redie.2017.19.1.765.

Peralta, A. (2007). Libro Blanco sobre Universidad y Discapacidad. Madrid: Real Patronato sobre Discapacidad.

Polo, M. T. (2017). Innovación para la formación en inclusión: actitudes de la comunidad universitaria. International Journal of Developmental and Educational Psychology, 4(1), 185-193.

Rodríguez-Martín, A. y Álvarez-Arregui, E. (2015). Estudiantes con discapacidad en la Universidad. Un estudio sobre su inclusión. Revista Complutense de Educación, 25(2), 457-479.

Sánchez-Palomino, A. (2011). La Universidad de Almería ante la integración educativa y social de los estudiantes con discapacidad: ideas y actitudes del personal docente e investigador. Revista de Educación, 354, 575-603.

Savage, L. B. y Wienke, W.D. (1989). Attitudes of secondary teachers toward mainstreaming. High School Journal, 73, 70-73.

Suriá, R., Bueno, A. y Rosser, A. (2010). Barreras mentales hacia los estudiantes con discapacidad: estudio de su evolución según la etapa formativa y la titulación. VII Congreso Iberoamericano de Psicología, Oviedo (julio, 2010).

Suria, R. (2012). Discapacidad e integración educativa: ¿qué opina el profesorado sobre la inclusión de estudiantes con discapacidad en sus clases? REOP 23(3), 96-109. 
Vaz, S., Wilson, N., Falkmer, M., Sim, A., Scott, M., Cordier, R. y Falkmer, T. (2015). Factors Associated with Primary School Teachers' Attitudes Towards the Inclusion of Students with Disabilities. PLoS One, 10(8). http://doi.org/10.1371/journal. pone.0137002. 